\title{
THE COEFFICIENTS OF STARLIKE FUNCTIONS
}

\section{J. A. HUMMEL ${ }^{1}$}

1. In his thesis [1] Bombieri shows that there exist constants $c_{n}$ such that if $f(z)=z+a_{2} z^{2}+\cdots$ is univalent in $|z|<1$ then

$$
\left|\operatorname{Re}\left(n-a_{n}\right)\right| \leqq c_{n} \operatorname{Re}\left(2-a_{2}\right) .
$$

The exact size of these coefficients is unknown. In his problem book [4, Problem 6.3] Hayman asked if there exist constants $d_{n}$ such that

$$
|n-| a_{n}|| \leqq d_{n}\left(2-\left|a_{2}\right|\right)
$$

for any $f$ in the class of normalized univalent functions in the unit disc.

The proof of the local maximum theory for the coefficients of univalent functions by Bombieri [2] and by Garabedian and Schiffer [3] gives strength to the conjecture that these $d_{n}$ exist, but no estimate of their size is available from these papers. However, we can make a precise estimate of the size of these coefficients for the subclass of the univalent functions consisting of the starlike functions. In this paper, we show that if $f(z)$ is starlike, then

$$
n-a_{n}=\gamma_{n}\left(2-a_{2}\right)
$$

where the $\gamma_{n}$ are constants depending on the function $f$ but satisfying

$$
\left|\gamma_{n}\right| \leqq n(n-1)(n+1) / 6
$$

for any starlike function. Furthermore, no better estimate than (4) is possible.

2. We denote by $S^{*}$ the class of starlike functions, that is functions which are regular in $U=\{z|| z \mid<1\}$, are normalized to have the series expansion $f(z)=z+a_{2} z^{2}+a_{3} z^{3}+\cdots$ and satisfy

$$
\operatorname{Re}\left\{z f^{\prime}(z) / f(z)\right\}>0
$$

in $U$ [7]. We denote by $K$ the class of close-to-convex functions [6], [8]. These are the functions which are regular in $U$ and which satisfy

$$
\operatorname{Re}\left\{z g^{\prime}(z) / f(z)\right\}>0
$$

in $U$ for some function $f \in S^{*}$. In this case we say that $g$ is close-to-

Received by the editors October 3, 1968.

${ }^{1}$ This work was supported in part by a grant from the National Science Foundation, NSF-GP 6891, to the University of Maryland. 
convex with respect to the starlike function $f$. If

$$
f(z)=b_{0}+b_{1} z+b_{2} z^{2}+\cdots
$$

is in the class $K$, then Reade [8] has shown that

$$
\left|b_{n}\right| \leqq n\left|b_{1}\right| \text {. }
$$

Let $\zeta$ be any point in $U$. Let

$$
\begin{aligned}
\Psi(z) & =(z-\zeta)\left(1-\zeta^{*} z\right) / z \\
& =-\zeta / z+\left(1+|\zeta|^{2}\right)-\zeta^{*} z
\end{aligned}
$$

Here, and throughout this paper, an asterisk on a complex quantity indicates the complex conjugate of that quantity. If $f \in S^{*}$ then

$$
\begin{aligned}
g(z) & =f(z) \Psi(z) \\
& =b_{0}+b_{1} z+b_{2} z^{2}+\cdots
\end{aligned}
$$

is univalent and weakly starlike as shown in [5]. The class of univalent weakly starlike functions clearly consists of functions mapping $U$ onto starshaped domains and hence forms a subclass of $K$. However, "clearly" is not the same as a proof. So let us prove

Lemma. 1. Let $f \in S^{*}$ and let $\Psi$ be defined by (7). Then $g(z)=f(z) \Psi(z)$ is close-to-convex with respect to $f$.

Proof. Let $r<1$. Define $f_{r}(z)=f(r z) / r$ and $g_{r}(z)=f_{r}(z) \Psi(z)$. Then $f_{r}(z)$ and $g_{r}(z)$ are regular for $|z| \leqq 1$. The same is true for the function

$$
z g_{r}^{\prime}(z) / f_{r}(z)=\left(z f_{r}^{\prime}(z) / f_{r}(z)\right) \Psi(z)+z \Psi^{\prime}(z) .
$$

Further, as $r \rightarrow 0, f_{r}(z) \rightarrow f(z), g_{r}(z) \rightarrow g(z)$, and $z g_{r}^{\prime}(z) / f_{r}(z) \rightarrow z g^{\prime}(z) / f(z)$, all uniformly in compact subsets of $U$.

Since $\Psi(z)$ is real and $z \Psi^{\prime}(z)=\zeta / z-\zeta * z$ is purely imaginary on $|z|=1$, and $z f_{r}^{\prime}(z) / f_{r}(z)=r z f^{\prime}(r z) / f(r z)$ has positive real part on $|z|=1$, it follows from (9) that $\operatorname{Re}\left\{z g_{r}^{\prime}(z) / f_{r}(z)\right\} \geqq 0$ for all $z \in U$. Therefore $\operatorname{Re}\left\{z g^{\prime}(z) / f(z)\right\} \geqq 0$ in $U$ also. A simple calculation shows that $z g^{\prime}(z) / f(z)=1-a_{2} \zeta+|\zeta|^{2}$ at $z=0$ and since $\left|a_{2}\right| \leqq 2$, it follows that $\operatorname{Re}\left\{z g^{\prime}(z) / f(z)\right\}>0$ at $z=0$. Hence $\operatorname{Re}\left\{z g^{\prime}(z) / f(z)\right\}>0$ for all $z \in U$ and the lemma is proved.

3. Once the above lemma has been proved, the remainder of the results follow quite easily. We prove first

ThEOREM. Let $f(z)=z+a_{2} z^{2}+a_{3} z^{3}+\cdots$ be in the class $S^{*}$. Then

$$
n-a_{n}=\gamma_{n}\left(2-a_{n}\right)
$$


where

$$
\gamma_{n}=\sum_{\nu=1}^{n-1} k_{n, \nu} \mu_{\nu}
$$

In this expression the $\mu_{\nu}$ are complex constants depending on $f$ but satisfying

$$
\mu_{1}=1, \quad\left|\mu_{\nu}\right| \leqq 1, \quad \nu=2,3, \cdots .
$$

The $k_{n, v}$ are constants independent of $f$ defined by

$$
k_{n, \nu}=\nu(n-\nu) \quad n=2,3, \cdots ; \nu=1,2, \cdots, n-1 .
$$

Finally, for every $n$

$$
\left|\gamma_{n}\right| \leqq n(n-1)(n+1) / 6 .
$$

Proof. From the lemma, the function $g(z)$ defined by $(8)$ is closeto-convex. It therefore satisfies (6). From (8),

$$
\begin{aligned}
& b_{1}=-\zeta a_{2}+\left(1+|\zeta|^{2}\right), \\
& b_{n}=-\zeta a_{n+1}+\left(1+|\zeta|^{2}\right) a_{n}-\zeta^{*} a_{n-1}, \quad n=2,3, \cdots .
\end{aligned}
$$

In these formulas we may let $a_{1}=1$ and $a_{0}=0$. Putting these values into (6) and dividing both sides by $\zeta$ gives

$$
\left|a_{n+1}-\left(1 / \zeta+\zeta^{*}\right) a_{n}+\left(\zeta^{*} / \zeta\right) a_{n-1}\right| \leqq n\left|a_{2}-\left(1 / \zeta+\zeta^{*}\right)\right| \text {. }
$$

This is true for every $n \geqq 1$ and each $\zeta \in U$. Letting $\zeta \rightarrow 1$, we have in the limit that

$$
\left|a_{n+1}-2 a_{n}+a_{n-1}\right| \leqq n\left|a_{2}-2\right|, \quad n=1,2,3, \cdots .
$$

This same result is equivalent to stating that, given $f \in S^{*}$, there exists a sequence of constants $\mu_{\nu}$ satisfying (12) such that

$$
a_{n+1}-2 a_{n}+a_{n-1}=n \mu_{n}\left(a_{2}-2\right) .
$$

Formula (10) is obvious in the case $n=2$. It follows, in general, by induction. Assuming (10) true for $n$ and $n-1$, it is easily verified from (15) that

$$
\begin{aligned}
(n+1)-a_{n+1} & =2\left(n-a_{n}\right)-\left[(n-1)-a_{n-1}\right]+n \mu_{n}\left(2-a_{2}\right) \\
& =\left[\sum_{\nu=1}^{n-1}\left(2 k_{n, \nu}-k_{n-1, \nu}\right) \mu_{\nu}-n \mu_{n}\right]\left(2-a_{2}\right) \\
& =\left[\sum_{\nu=1}^{n} k_{n+1, \nu} \mu_{\nu}\right]\left(2-a_{2}\right) .
\end{aligned}
$$


This proves (10).

Inequality (14) follows from (11) and the fact that

$$
\sum_{\nu=1}^{n-1} k_{n, \nu}=\sum_{\nu=1}^{n-1} \nu(n-\nu)=n(n-1)(n+1) / 6
$$

as easily verified by induction.

4. Inequality (14) is sharp in the sense that no better numerical estimate is possible. This follows from the following

LEMMA 2. Let $\kappa=e^{i \alpha}$, and set

$$
h(z)=z /(1-\kappa z)\left(1-\kappa^{*} z\right)=z+a_{2} z^{2}+a_{3} z^{3}+\cdots .
$$

Then $h(z) \in S^{*}$ and is such that for each $n \geqq 2$

$$
\lim _{a \rightarrow 0} \frac{n-a_{n}}{2-a_{2}}=n(n-1)(n+1) / 6 .
$$

Proof. From (16)

$$
\begin{aligned}
a_{2} & =\kappa+\kappa^{*}=2 \cos \alpha \\
a_{n} & =\kappa^{n-1}+\kappa^{*} \kappa^{n-2}+\cdots+\kappa^{*^{n-2}} \kappa+\kappa^{*^{n-}} \\
& =\left(\kappa^{n}-\kappa^{*^{n}}\right) /\left(\kappa-\kappa^{*}\right) \\
& =\sin n \alpha / \sin \alpha .
\end{aligned}
$$

Therefore

$$
\begin{aligned}
\frac{n-a_{n}}{2-a_{2}} & =\frac{n \sin \alpha-\sin n \alpha}{2 \sin \alpha(1-\cos \alpha)} \\
& =\frac{n \alpha-(n / 3 !) \alpha^{3}-n \alpha+\left(n^{3} / 3 !\right) \alpha^{3}+o\left(\alpha^{3}\right)}{2[\alpha+o(\alpha)]\left[\alpha^{2} / 2 !+o\left(\alpha^{2}\right)\right]} \\
& =n(n-1)(n+1) / 6+o(1) \quad \text { as } \quad \alpha \rightarrow 0,
\end{aligned}
$$

which is the desired result (17).

Since the starlike functions form a subclass of the normalized univalent functions, the results of this paper give $n(n-1)(n+1) / 6$ as a lower bound for the constants $c_{n}$ and $d_{n}$ in (1) and (2). The writer is convinced that the constants $d_{n}$ are actually much larger than this (assuming that they exist) and feels that it is probable that the $c_{n}$ are larger also, but has no firm evidence on which to base this assertion.

I thank the referee for his suggestion of a shorter proof at one 
point and for pointing out some statements in the original version whose meanings were not very clear.

\section{REFERENCES}

1. Enrico Bombieri, Sull'integrazione approssimata dell'equazione differenziale di Löwner e le sue applicazioni alla teoria delle funzioni univalenti, Thesis, Università di Milano, Milano, 1963.

2. - On the local maximum property of the Koebe function, Invent. Math. 4 (1967), 26-67.

3. P. R. Garabedian and M. Schiffer, The local maximum theorem for the coefficients of univalent functions, Arch. Rational Mech. Anal. 26 (1967), 1-32.

4. W. K. Hayman, Research problems in function theory, The Athlone Press, London, 1967.

5. J. A. Hummel, Multivalent starlike functions, J. Analyse Math. 18 (1967), 133160.

6. Wilfred Kaplan, Close-to-convex schlichtfunctions, Michigan Math. J. 1 (1953), 169-185.

7. R. Nevanlinna, Über die konforme Abbildung von Sterngebieten, Öfvres. Finska Vet. Soc. Förh. 53 (A) (1921), Nr. 6.

8. M. O. Reade, On close-to-convex univalent functions, Michigan Math. J. 3 (1955), $59-62$.

9. W. Rudin, Real and complex analysis, McGraw-Hill, New York, 1966.

UNIVERSITY OF MARYLAND 